\title{
EXTRAÇÃO E PRÉ-TRATAMENTO QUÍMICO DE FRUTANOS DE YACON,
}

\author{
Polymnia sonchifolia
}

\author{
Cláudio CABELLO
}

\section{RESUMO}

O processo de desintegração de tubérculos de yacon promove a incorporação de oxigênio que favorece a oxidação de compostos fenólicos e decorrente contaminação do extrato aquoso com cores e odores objetáveis que prejudicam a sua utilização. Este estudo buscou verificar uma metodologia simplificada de extração dos carboidratos presentes nos tubérculos de yacon e efetuar uma primeira remoção de resíduos e compostos originários das cores e odores indesejáveis, utilizando processos de coagulação e precipitação, aplicando tecnologias de baixo custo. Após a etapa de trituração e remoção dos restos celulares, o extrato teve seu pH aumentado para 9,5 e a temperatura para $90^{\circ} \mathrm{C}$, seguido de sedimentação. A remoção deste precipitado foi realizada por filtração em papel após a coagulação com diferentes concentrações de sulfato de alumínio comercial. Verificou-se que a melhor concentração de coagulante no extrato foi de $100 \mathrm{ppm}$, removendo 90,6\% dos compostos coloridos. O balanço de massa mostrou uma recuperação de $47,5 \%$ dos sólidos totais sendo que $35,6 \%$ estão na forma de carboidratos, verificado através da concentrção de carbono orgânico. O processo de pré-tratamento químico causou hidrólise nos oligofrutanos, detectado por um aumento na concentração de açúcares redutores totais, diretamente proporcional à concentração de sulfato de alumínio utilizado no tratamento de coagulação. Análises cromatográficas indicaram o perfil dos açúcares no hidrolisado e a extensão desta hidrólise que foi considerada pequena. A metodologia aplicada mostrou-se fácil, de baixo custo, eficiente e aplicável em agroindústrias nas zonas produtoras desta espécie de raiz tropical.

Palavras-chave: yacon; inulinas; oligofrutossacarídeos; separação; coagulação.

\section{SUMMARY}

EXTRACTION AND CHEMICAL PRE-TREATMENT OF FRUCTANS FROM YACON, Polymnia sonchifolia. The process of disintegration of yacon roots promotes the oxygen incorporation that favors the phenolic oxidation and current contamination of the aqueous extract with undesirable colors and scents that damage its use. This work aims at verifying the simplified methodology of extraction of the prejent carbohydrates in the yacon roots and to make a first removal of residues and undesirable scents, using coagulation processes and precipitation applying low cost technologies. After the grinding stage and removal of cellular remains, the extract had increased by $\mathrm{pH} 9.5$ and the temperature $90^{\circ} \mathrm{C}$ following sedimentation. The removal of the precipitat was accomplished by filtration in paper after coagulation with different concentrations of commercial aluminium sulfate. It was verified that the best coagulant concentration in the extract was of $100 \mathrm{ppm}$, removing $90.6 \%$ of the colored compositions. The mass balance showed a recovery of $47.5 \%$ of the total solids and $35.6 \%$ in the carbohydrates shape, verified through the concentration of the organic carbon. The process of chemical pré-treatment caused hydrolyses in the oligofrutanos detected directly by an increase in the concentration of reduces total sugar proportional for concentration of aluminium sulfate used in the coagulation treatment. Chromatography analyses showed the profile of the sugars in the hydrolysate . The extension of this hydrolyses was considered small. The applied methodology was shown to be easy, of low cost, efficient and applicable in agroindustries in the zones producing this species of tropical roots.

Keywords: yacon; inulin; oligofructan; separation process; coagulation.

\section{1 - INTRODUÇÃO}

Oyacon é uma espécie da família Asteraceae originária das regiões andinas e extremamente adaptável quanto ao clima, altitude e tipo de solo e encontra utilização principalmente na alimentação humana [11]. Produz tubérculos subterrâneos suculentos que são ricos em frutanos e fibras não digeriveis e atingem uma produtividade relatada de 120 toneladas por hectare de raízes tuberosas [7]. Os frutanos são carboidratos de reserva na forma de polímeros de D-frutose unidos por ligações tipo $\beta(2 \rightarrow 1)$ e tendo uma glicose na extremidade da cadeia. Devido à acumulação de frutanos em seus tubérculos, o yacon tem apresentado potencialidades como fonte des-

Recebido para publicação em 26/11/2001. Aceito para publicação em 28/04/2005(000781).

CERAT/Universidade Estadual Paulista. Caixa Postal 237. CEP: 18603-970,Botucatu/SP.E-mail:dircerat@fca.unesp.br

A quem a correspondência deve ser enviada. tes biopolímeros para utilização nutricional e terapêutica $[4,10]$.

Outras fontes importantes de frutanos são a Chicorium intybus (chicória), Dahlia pinuata (dália) e Helianthus tuberosus (alcachofra de Jerusalém), que possuem este biopolímero de 10 a 14, 20 e 6 unidades de monossacarídeos, respectivamente [14]. A frutose, como adoçante natural, apresenta vantagens sobre a sacarose e glicose, sendo 1,3 vezes mais doce e menos cariogênica que a sacarose. A ingestão de uma quantidade normal de frutose por humanos não depende de insulina, sendo também apropriada para consumo por diabéticos e pessoas em dieta com baixas calorias. Além disso, por cristalizar menos facilmente que a sacarose, proporciona textura cremosa em doces e alimentos congelados, preserva o "flavor" dos alimentos, realçando o aroma de frutas e de alimentos vegetais $[3,20]$.

O escurecimento oxidativo de frutas é catalisado pelas enzimas do grupo das polifenoloxidases e resulta da oxidação de fenóis e eventual polimerização não enzimática das quinonas formadas em taninos ou melaninas [1]. 
Esta oxidação se dá em presença de oxigênio livre, escurecendo rapidamente a superfície recém-cortada dos tubérculos, prejudicando sua aparência e a de seus produtos. De acordo com BOTREL \& CARVALHO [1] a peroxidase tem uma função importante na biossíntese de lignina e também está associada ao mecanismo de resistência de algumas plantas. A função mais importante da polifenoloxidase é a capacidade de oxidar inicialmente monofenóis para o-difenóis (atividade cresolásica), seguida por uma oxidação de o-difenóis para o-quinona (atividade catecolásica). Ambas as reações utilizam oxigênio molecular e os pigmentos escuros formados pela oxidação de o-quinonas não contém nitrogênio e, portanto, são diferentes das melaninas.

Os fatores responsáveis pela reação são as enzimas, o substrato e o oxigênio e, teoricamente, a interferência em um desses fatores impede a reação de ocorrer, controlando assim a oxidação. Do ponto de vista prático, o controle do escurecimento enzimático é geralmente limitado à inibição da enzima utilizando o calor, pois inativa-se as enzimas polifenoloxidase e peroxidase, responsáveis pela reação de escurecimento.

Os processos de extração dos frutanos realizam-se sob atmosfera livre, devido à viabilidade econômica e à ocorrência de reações de oxidações indesejáveis são minimizadas pelo controle de temperatura e $\mathrm{pH}$ aplicado aos extratos, limitando a ação enzimática. Ainda assim se faz necessário um posterior processo para remoção dos compostos responsáveis pelo escurecimento.

A separação de sólidos dispersos em fase líquida envolve etapas de sedimentação, filtração, flotação e/ou centrifugação que, dentre outros fatores, é afetada fundamentalmente pelo tamanho da partícula do sólido em suspensão. Tratamentos quimicos buscam aumentar o tamanho destes sólidos, de modo a otimizar as separações do ponto de vista de rapidez, eficiência e rendimento [2].

Partículas em suspensão apresentam cargas superficiais que definem atração/repulsão em relação às outras, de modo que, sistemas podem ser montados para que substâncias químicas alterem estes estados de equilíbrio eletrostático e produzam atrações que resultam na formação de flocos mais facilmente sedimentáveis [12]. Segundo MOODY [9], pré-tratamentos químicos que objetivam a separação de fases sólidos/liquidos através da coagulação e/ou floculação, estão diretamente ligados à natureza da substância a ser separada para se obter a máxima eficiência e à menor degradação possivel e isto pode ser verificado experimentalmente.

Dentre os vários produtos químicos de baixo custo utilizados para floculação, o sulfato de alumínio é o mais extensivamente utilizado, principalmente em sistemas de produção e tratamento de água potável. Em solução aquosa o sulfato consome alcalinidade e produz um complexo de hidróxido de alumínio com atividade de superficie, que atrai particulas de cargas positivas formando flocos e, deste modo, os remove da suspensão. Se por um lado remove contaminantes clarificando soluções, de outro acrescenta sais inorgânicos na água, de modo que a sua utilização deve ser indicada a situações específicas avaliando custo/beneficios.

Este estudo busca verificar uma metodologia simplificada de extração dos frutanos presentes nos tubérculos de yacon e efetuar uma primeira remoção do resíduo e dos compostos originários das cores e odores indesejáveis, utilizando processo de coagulação e precipitação com sulfato de alumínio comercial.

\section{2 - MATERIAL E MÉTODOS}

\section{1 - Material botânico}

Foram utilizadas raízes retiradas de plantas de yacon cultivadas nos canteiros experimentais do CERAT, localizado na Fazenda Experimental do Lageado em Botucatu/SP. Foram estocadas em sacos de PVC em refrigerador a $5^{\circ} \mathrm{C}$ e utilizadas em até 7 (sete) dias após a coleta.

\section{2 - Produção dos extratos}

O extrato foi obtido por uma seqüência de operações que, basicamente, visavam a separação de sólidos de uma fase líquida por processos físicos e químicos.

\subsection{1 - Desintegração das raízes e separação}

Foram tomadas $400 \mathrm{~g}$ de raizes de yacon que foram rapidamente cortadas em toletes e colocadas num copo de liquidificador contendo $200 \mathrm{~g}$ de água destilada aquecida a $90^{\circ} \mathrm{C}$. O tempo de desintegração foi de 2 minutos em velocidade máxima. A seguir, todo o material foi transferido para um funil tipo Buchner contendo 2 folhas de papel de filtro quantitativo e filtrado à pressão negativa. Após esgotamento do extrato, o resíduo foi cuidadosamente lavado com $300 \mathrm{~mL}$ de água destilada a $90^{\circ} \mathrm{C}$, para lixiviação dos carboidratos ainda absorvidos nos restos do material celular. Este bolo filtrante foi estocado para realização de outras avaliações.

\subsection{2 - Tratamento térmico e filtração}

Ao extrato, foram adicionados $\mathrm{NaOH} 1 \mathrm{M}$ até que o $\mathrm{pH}$ ficasse entre 9,5 a 9,8 e colocado em banho-maria a $95^{\circ} \mathrm{C}$ por 20 minutos, de modo a coagular as proteínas, gomas e matérias graxas. A seguir, todo o material foi descarregado num funil tipo Buchner contendo 2 folhas de papel de filtro qualitativo e filtrado à pressão negativa. $\mathrm{O}$ extrato foi estocado para avaliações posteriores.

\section{3 - Determinação da granulometria do resíduo}

Uma amostra úmida de $100 \mathrm{~g}$ retirada do bolo filtrado foi colocada num conjunto de peneiras previamente pesadas, com malhas de 0,$85 ; 0,425 ; 0,300 ; 0,212 ; 0,150$; 0,090 e a última de $0,045 \mathrm{~mm}$ de abertura. A seguir, um fluxo de água corrente foi colocado sobre a primeira peneira de 20 e a água foi lixiviando os particulados que foram sendo retidos nas peneiras inferiores. Por diferença 
de peso calculou-se as proporções retidas nas diversas peneiras.

\section{4 - Processo de pré-tratamento do extrato}

Amostras de $50 \mathrm{~mL}$ do extrato foram dispostas em frascos erlenmeyer de $125 \mathrm{~mL}$ e em cada conjunto de 5 frascos foram adicionados $1 \mathrm{~mL}$ de solução de sulfato de alumínio, de modo que, a concentração final ficasse com 50, 100, 150 e 200ppm de sulfato de alumínio. Um frasco recebia equivalente volume de água destilada e seria o testemunha. Os frascos foram agitados fortemente no momento da adição e, após um tempo de 15 minutos, o conteúdo foi filtrado à gravidade em papel de filtro qualitativo. O extrato filtrado foi coletado em frasco e estocado em geladeira a $5^{\circ} \mathrm{C}$. O mesmo procedimento para tempos de 30 e 45 minutos.

\section{5 - Determinação da cor}

A verificação dos efeitos de remoção dos contaminantes que caracterizam a eficiência dos processos de adsorção foram avaliados através de espectrofotometria de absorção molecular utilizando a metodologia proposta pela SOCIETY OF SOFT DRINK TECHNOLOGISTS [17], que produz resultados numa escala arbitrária denominada RBU:

$$
\operatorname{cor}(\mathrm{RBU})=\frac{\left(\mathrm{A}_{420 \mathrm{~mm}}-2 \times \mathrm{A}_{720 \mathrm{~mm}}\right) \times 1000}{\text { cel. }(\mathrm{cm}) \times \text { Sól. }(\mathrm{g} / \mathrm{mL})}
$$

Foi utilizado um espectrofotometro Varian modelo Cary 50 Bio com detector tipo diodo array que escaneava o espectro desde 200 até $900 \mathrm{~nm}$, e os valores de absorção em 420 e $720 \mathrm{~nm}$ eram registrados para posteriores cálculos.

\section{6 - Determinação da concentração de carbo- no orgânico total}

Foi utilizado um cromatógrafo Shimatzu modelo TOC 5000A com detector ajustado para medir concentrações de $\mathrm{CO}_{2}$ emitida pela calcinação da matéria orgânica dos extratos.

\section{7 - Determinação da concentração de açúcares}

Foi utilizado um cromatógrafo líquido de alta performance (HPLC) marca Varian modelo ProStar, detector $350 \mathrm{RI}$, água como fase móvel a $0,8 \mathrm{~mL} / \mathrm{min}$, temperatura $80^{\circ} \mathrm{C}$ e coluna da Biorad Aminex modelo HPX$87 \mathrm{P}$. O cromatograma indica o perfil dos diversos sacarídeos na amostra e suas concentrações relativas. A concentração de açúcares redutores utilizou a metodologia de SOMOGY [15].

\section{8 - Determinação da concentração de cinzas}

Foi medido gravimetricamente após calcinação das amostras em forno mufla a $550^{\circ} \mathrm{C}$, por 4 horas [6].

\section{3 - RESULTADOS E DISCUSSÃO}

A inulina apresenta solubilidade somente em água quente [19] e, por este motivo, na operação de desintegração das raízes utilizou-se desta propriedade para propiciar tanto a formação de uma suspensão com maior ponto de fluidez, como para lixiviação deste polissacarídeo do interior das células fragmentadas. A agitação vigorosa promoveu uma maior incorporação de oxigênio ao meio e disto resultou um maior escurecimento, como resultado da oxidação de fenóis e eventual polimerização não enzimática das quinonas formadas em taninos ou melaninas [1]. Este tipo de desintegração fragmenta extensivamente as células do yacon e possibilita a liberação das inulinas de interesse. A desvantagem do método seria o escurecimento enzimático, ativado pela incorporação de oxigênio do ar atmosférico e a mistura de oligo e polifrutanos que seriam evitadas na exaustiva metodologia proposta por POLLOCK \& JONES [13], mas por outro lado é rápida e eficiente.

O processo de desintegração reduziu a estrutura das raízes e a distribuição das partículas após peneiramento a úmida, estão dispostos na Tabela 1. Foi verificado que, a raiz possuía umidade total de $83,9 \%$ e foi necessária a adição de pequeno volume de água para auxiliar a desintegração mecânica. O restante da água foi utilizado para lixiviar do material triturado, previamente filtrado, o extrato contendo as moléculas de interesse.

TABELA 1 - Dimensões do material particulado resultante do processo de desintegração das raízes de yacon

\begin{tabular}{ccc}
\hline Peneira TYLER & Abertura malha $(\mathrm{mm})$ & Material retido $(\%)$ \\
\hline 20 & 0,850 & 39,1 \\
35 & 0,425 & 23,9 \\
48 & 0,300 & 11,8 \\
65 & 0,212 & 14,1 \\
100 & 0,150 & 6,7 \\
170 & 0,090 & 1,7 \\
- & 0,045 & 2,7 \\
\hline
\end{tabular}

O tratamento térmico e a adição de álcalis no extrato objetivou a precipitação de substâncias coloidais formadas em sua maior parte por proteínas e matérias graxas. A temperatura e o $\mathrm{pH}$ alcalino modificam a configuração estrutural das proteinas e por decorrência, a cor do extrato modificou-se, e o precipitado, de consistência gelatinosa, aderiu à superficie interna do vaso de contenção. Uma segunda filtração com papel de filtro removeu estes precipitados. Foram realizadas medidas dos produtos originados das etapas de processamento que permitiram efetuar o balanço de massa, expresso na Figura 1.

A remoção das substâncias coloidais remanescentes após um primeiro tratamento térmico e precipitação, seria complementada com utilização do sulfato de alumínio, que auxiliou o processo de aglutinação de substâncias coloidais e formação de flocos mais facilmente filtráveis nas amostras de extratos submetidas ao tratamento [9]. SRIKANTA et al [16], compararam a eficiência de vários agentes desproteinizantes aplicados a hidrolisados 


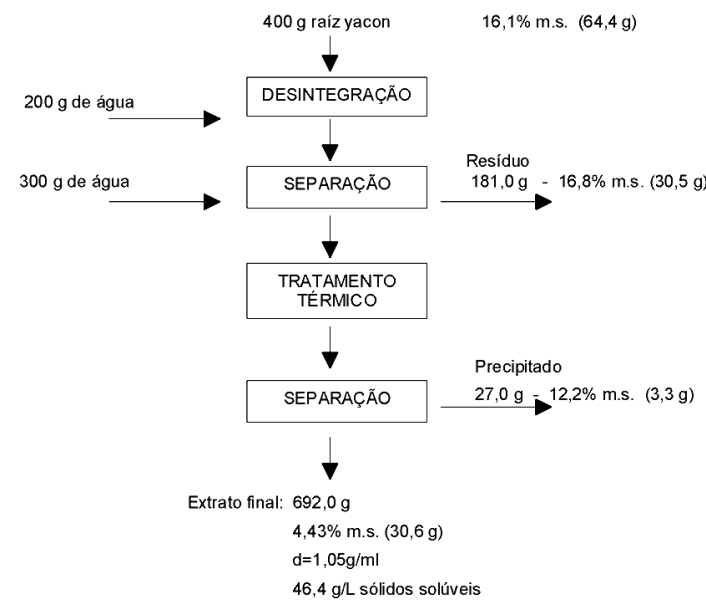

FIGURA 1 - Balanço de massa verificado na metodologia utilizada

de resíduos da industrialização da mandioca e verificaram que o caulin foi o mais econômico. Aplica $5 \% \mathrm{p} / \mathrm{v}$ com tempo de contato de 10 minutos a $25^{\circ} \mathrm{C} \mathrm{em} \mathrm{pH} 1,0-$ 1,5 e complementa com aplicação de carvão ativado.

Na Tabela 2 verifica-se que o melhor resultado foi em amostras de extrato com 100ppm de sulfato de alumínio que produziu uma cor de 3.758,7 unidades, sendo que o mais indicado tempo de contato para floculação foi de 15 minutos. Considerando-se o extrato inicial, a remoção dos contaminantes responsáveis pela cor, foi de $90,6 \%$. Os tempos de contato da reação de 15,30 e 45 minutos não produziram diferenças significativas entre si na remoção de cores, e portanto, o tempo de 15 minutos foi considerado como adequado.

O sulfato de alumínio reage com a alcalinidade existente na água e forma o complexo de hidróxido de alumínio que, desestabilizando eletricamente o sistema, provoca a aglutinação das substâncias coloidais dispersas que produzem cores e aromas indesejáveis na suspensão [8]. A alcalinidade disponivel nas amostras, foi suficiente para produzir a complexação do sulfato de alumínio, gerando o hidróxido necessário à atividade como agente aglutinante.

Na Tabela 3, observam-se os valores de açúcares redutores totais e a concentração de carboidratos totais verificados nas amostras submetidas a diferentes concentrações de sulfato de alumínio, após um tempo de contato de 15 minutos.

Verifica-se por estes valores, que as perdas relativas ao processo de remoção de contaminantes coloidais produziram diminuição na concentração de carbono orgânico, indicando a presença de carboidratos nestes materiais removidos. O proporcional aumento na concentração de açúcares redutores, deveu-se à hidrólise de ligações existentes entre os monômeros frutose dos frutanos, favorecida pelo baixo $\mathrm{pH}$ do meio, conseqüência das reações de complexação do sulfato de aluminio a hidróxido de alumínio.
TABELA 2 - Valores de absorbância a 420 e 720nm, concentração de sólidos e unidades de cor das amostras tratadas com diferentes concentrações de $\mathrm{Al}_{2}\left(\mathrm{SO}_{4}\right)_{3}$, com tempo de contato de 15 minutos

\begin{tabular}{ccccc}
\hline $\begin{array}{c}\text { Concentração } \mathrm{Al}_{2}\left(\mathrm{SO}_{4}\right)_{3} \\
(\mathrm{ppm})\end{array}$ & $\begin{array}{c}\mathrm{Abs} .420 \mathrm{~nm} \\
(\mathrm{UA})\end{array}$ & $\begin{array}{c}\mathrm{Abs} .720 \mathrm{~nm} \\
(\mathrm{UA})\end{array}$ & $\begin{array}{c}\text { Conc. Sólidos } \\
(\mathrm{g} / \mathrm{ml})\end{array}$ & $\begin{array}{c}\text { Cor } \\
\text { (Unidade) }\end{array}$ \\
\hline 0 & 4,4947 & 1,5542 & 0,0334 & $41.505,9$ \\
50 & 2,9796 & 0,6053 & 0,0327 & $54.097,8$ \\
100 & 0,1390 & 0,0088 & 0,0315 & $3.758,7$ \\
150 & 0,2016 & 0,0140 & 0,0319 & $5.442,0$ \\
200 & 0,1688 & 0,0121 & 0,0323 & $4.476,7$ \\
\hline
\end{tabular}

TABELA 3 - Concentração de açúcares redutores totais, de carboidratos totais, carbono orgânico total, cinzas e pH das amostras tratadas com diferentes concentrações de hidróxido de aluminio

\begin{tabular}{cccccc}
\hline $\begin{array}{c}\text { Concentração } \mathrm{Al}_{2}\left(\mathrm{SO}_{4}\right)_{3} \\
(\mathrm{ppm})\end{array}$ & $\begin{array}{c}\text { Açúcares } \\
\text { redutores } \\
(\mathrm{g} / \mathrm{l})\end{array}$ & $\begin{array}{c}\text { Carbono } \\
\text { orgânico total } \\
(\mathrm{ppm})\end{array}$ & $\begin{array}{c}\text { Carboidratos } \\
\text { totais } \\
(\mathrm{g} / \mathrm{l})\end{array}$ & $\begin{array}{c}\text { Cinzas } \\
(\%)\end{array}$ & $\mathrm{PH}$ \\
\hline 0 & 19,9 & 14.844 & 37,1 & 0,16 & 9,50 \\
50 & 20,8 & 14.524 & 36,3 & 0,19 & 4,30 \\
100 & 21,3 & 14.012 & 35,0 & 0,22 & 3,80 \\
150 & 21,7 & 14302 & 35,7 & 0,25 & 3,50 \\
200 & 21,8 & 14.366 & 35,9 & 0,28 & 3,30 \\
\hline
\end{tabular}

Os cromatogramas da Figura 2 mostram os perfis dos açúcares das amostras de extrato de yacon antes e após o tratamento e, na Tabela 4, as porcentagens relativas destes açúcares. A remoção dos contaminantes produziu uma diminuição na concentração dos carboidratos, com um aumento nas concentrações relativas de frutanos e sacarose. Isto, provavelmente, seja devido à hidrólise de frutanos de alto peso molecular, que a resolução da cromatografia (coluna e detector) não pôde quantificar. Se a utilização dos extratos destinar-se a alimentos prebióticos, este processo apresenta desvantagens devido ao fato desta hidrólise diminuir a concentração dos polissacarídeos [5]. Modificações químicas em frutanos para utilizações mais específicas, também requerem concentrações de polissacarídeos de maior grau de polimerização [18] .

O cromatograma da Figura 2, mostra que os frutanos representam $20,8 \%$ do conteúdo dos sólidos solúveis no extrato da amostra de yacon estudada, enquanto que frutose atinge quase que a metade destes compostos.

As análises para verificação da variação de contaminantes inorgânicos aitados ao extrato, por conta da adição do sulfato de alumínio, indicaram um aumento no teor de cinzas passando de 0,16 para $0,22 \%$, ou seja, um aumento de $37,5 \%$. Apesar do significativo aumento relativo, o valor absoluto pode ser considerado razoável, visto que, um típico hidrolizado enzimático de glicose contém um teor de cinzas em torno de $0,3 \%$, mas que, pode ser reduzido a menos de $0,02 \%$ com aplicação em colunas trocadoras de íons [10]. 


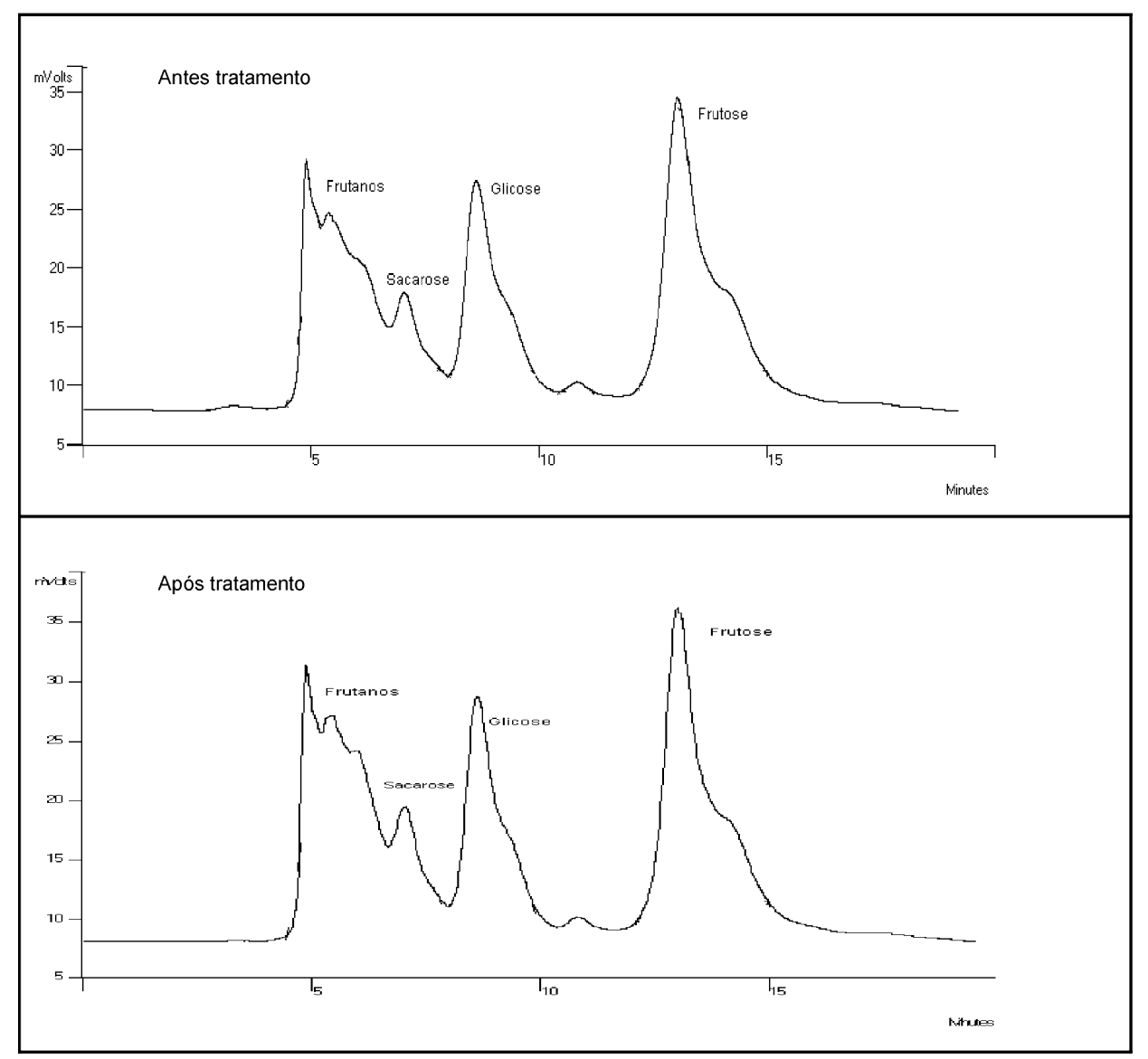

FIGURA 2 - Os cromatogramas mostram os perfis dos sacarídeos nos extratos antes e após o tratamento de remoção de contaminantes coagulados com o sulfato de alumínio $100 \mathrm{ppm}$ e tempo de floculação de 15 minutos

TABELA 4 - Porcentagens dos açúcares nas amostras do extrato antes e após tratamento com 100 ppm de sulfato de aluminio e tempo de contato de 15 minutos

\begin{tabular}{lccc}
\hline Açúcares & Antes tratamento (\%) & Após tratamento (\%) & Diferença (\%) \\
& & & \\
\hline Frutanos & 18,8 & 20,8 & 2,0 \\
Sacarose & 7,4 & 8,2 & 0,8 \\
Glicose & 24,9 & 23,8 & 1,1 \\
Frutose & 48,3 & 46,6 & 1,7 \\
Outros monossac. & 0,6 & 0,6 & 0 \\
& 100,0 & 100,0 & \\
\hline
\end{tabular}

WENLING, HUIYING \& SHIYUAN [21], utilizaram processo contínuo enzimático para obter xaropes de frutose a partir de inulinas extraídas de alcachofra de Jerusalém, que apresenta concentração de $80 \%(\mathrm{p} / \mathrm{p})$ base seca deste polissacarídeo em suas raízes. Considerando esta como sendo a utilização, a variedade de yacon estudada poderia ser bem explorada; mas, se o objetivo for recuperar frutanos de pequenas cadeias para utilização prebiótica, então não seria adequado, em face da concentração de frutanos estar em aproximadamente $9,9 \%(\mathrm{p} / \mathrm{p})$ base seca nas raízes.

\section{4 - CONCLUSÕES}

O processo de extração utiliza operações de separação sólido/líquido em duas etapas bem características; a primeira separando resíduos vegetais e a segunda removendo gomas, proteínas, graxas, etc. e, em ambas, a operação de separação foi em papel de filtro de baixo custo e simplicidade de utilização. O pré-tratamento químico com adição de sulfato de alumínio provocou alterações que possibilitaram a agregação de particulados e desta maneira foi possivel efetuar a separação da fase líquida por processo de filtração simples.

O processo utilizado mostrou-se simplificado e de fácil realização, causando pequenas repercussões nos compostos de interesse existente no extrato.

\section{5 - REFERÊNCIAS BIBLIOGRÁFICAS}

[1] BOTREL, N; CARVALHO,V.D. Efeito do Peso do Fruto no Escurecimento Interno e Qualidade do Abacaxi. Atividade de Polifenoloxidase, peroxidase e compostos fenólicos. Pesquisa Agropecuária Brasileira, v. 28, n. 6 , p. 733-742, 1993.

[2] COULSON, J.M.; RICHARDSON, J.F. 1984. Ingenieria Quimica, 3a. ed., v. III, Editorial Reverte: Barcelona. 
[3] GENNARO, S., BIRCH, G.G., PARKE, S.A., STANCHER, B. Studies on the physicochemical properties of inulin and inulin oligomers. Food Chemistry, v. 68, p. 179183, 2000.

[4] GIBSON, G.R., ROBERFROID, M.B. Dietary modulation of the human colonic microbiota: introducing the concept of prebiotic. Journal Nutrition, v. 125, p. 1401-1412, 1995.

[5] GUPTA, A K., KAUR, M., SINGH, R. Potencial medicinal and nutritional uses of chicory roots and inulin. In: FUCHS, A. (ed.) Inulin and inulin-containing crops, $1^{\text {a }}$ Elsevier: Amsterdam, 1993. p.359-365.

[6] IAL Normas Analiticas do Instituto Adolfo Lutz, $3^{\mathrm{a}}$ ed., São Paulo: Instituto Adolfo Lutz, 1985. 533p. (Volume 1, Métodos Químicos e Físicos para Análises de Alimentos).

[7] KAKIHARA,T.S., CÂMARA, F.L.A., VILHENA, S.M.C. et al. Cultivo e industriaalização de yacon (Polymnia sonchifolia): uma experiência brasileira. I Congresso Latino Americano de Raizes Tropicais e IX Congresso Brasileiro de Mandioca, São Pedro, SP, resumo 148, 1996.

[8] METCALF \& EDDY Chemical Unit Process in: Wastewater Engineering: Treatment, Disposal and Reuse, $2^{\mathrm{a}}$ ed. Boston: McGraw-Hill, 1979, 258-310p.

[9] MOODY, G.M. Pre-Treatment Chemical. Filtration \& Separation, v.4, p. 328-36, 1995.

[10] MULVIHILL, P.J. Crystalline and liquid dextrose products:Production, properties and applications in: SCHENCK, F.W., HEBEDA, R.E. (Ed.) Starch Hydrolysis Products, $1^{\text {a }}$ ed.New York:VCH Publisher, 1992. Cap.5, p.121-176.

[11] NATIONAL RESEARCH COUNCIL 1989. Lost crops of the incas: little-known plants of the Andes whit promise for worldwide cultivation. National Academy Press- Washington.

[12] OTHMER, K. Flocculating agents in: OTHMER, K. Encyclopedia of Chemical Technology. v.10, $3^{\text {a }}$ ed. New York: Willey Interscience, 1979, 489p.
[13] POLLOCK,C.J., JONES, T. Seasonal patterns of fructan and metabolism in forage grasses. New Phytologie, v.83, p.8-15, 1979.

[14] PRAZNIK, W., BAUMGARTNER, S., HUBER, A. Determination of molecular composition of inulin by means of enzymatic and chromatographic methods. J. Chromatogr., v. 303, p. 417, 1994.

[15] SOMOGY, M. Determination of blood sugar. J. Biol. Chem. n. 160, p. 69-73, 1945.

[16] SRIKANTA, S., JALEEL, S.A., KUNHI,A.A.M., REHANA, F., GHILDYAL, N.P., LONSANE, B.K., KARANTH, N.G. Downtream processing of the hydrolysate from cassava fibrous wate in the production of confectioner's syrup. Starch, v. 41, p. 196-200, 1989.

[17] SSDT - Society of Soft Drink Technologists. Guidelines and Methodes for HFCS-42 and HFCS-55 for Bottlers, 1988.

[18] Stevens, C.V., Meriggi, A, Booten, K. Chemical modification of inulin, a valuable renewable resource, and its industrial applications. Biomacromolecules, v. 2, n. 1, p. 1-16, 2001.

[19] VENDRELL-PASCUAS,S. , CASTELLOTE-BARGALLÓ, A.I., LOPEZ-SABATER, M.C. Determination of inulin in meat products by high-performance liquid chromatography with refractive index detection. Journal of Chromatography A., v.881, p.591-597, 2000.

[20] YAMAZAKI, H.; MATSUMOTO, K. Production of fructooligosaccharide-rich fructose syrup. In: FUCHS, A (Ed.) Inulin and inulin-containing crops, $1^{\underline{a}}$ ed. Elsevier: Amsterdam, p. 355-357, 1993.

[21] Wenling, W., HUiYing, W.W.L, ShiYUAN, W. Continuous preparation of fructose syrups from Jerusalem artichoke tuber using immobilized intracellular inulinase from Kluveromyces sp. Y-85. Process Biochemistry, v. 34, p. 643-646, 1999. 